\title{
Modified HE4, CA125, and ROMA cut-off values and predicted probability of ovarian tumor in Chinese patients
}

\author{
Xingping Zhao, Meidan Zhao, Bingsi Gao, Aiqian Zhang, Dabao Xu \\ Department of Gynecology, The Third Xiangya Hospital of Central South University, Changsha, China \\ Contributions: (I) Conception and design: D Xu, A Zhang; (II) Administrative support: D Xu; (III) Provision of study materials or patients: D Xu; (IV) \\ Collection and assembly of data: X Zhao, M Zhao; (V) Data analysis and interpretation: X Zhao, B Gao; (VI) Manuscript writing: All authors; (VII) \\ Final approval of manuscript: All authors. \\ Correspondence to: Dabao Xu and Aiqian Zhang. Department of Gynecology, the Third Xiangya Hospital of Central South University, 138 Tongzipo \\ Rd, Changsha 410013, China. Email: forxudabao@126.com; 513113464@qq.com.
}

\begin{abstract}
Background: Most prior studies investigating the risk of ovarian malignancy algorithm (ROMA) with cancer antigen 125 (CA125) and human epididymis protein 4 (HE4) have involved Caucasian population or other populations. To date, there have been no unique calculations of predicted probability (PP) risk specifically for Chinese populations to help physicians in primary care settings.
\end{abstract}

Methods: A group of 534 women with ovarian tumor diagnoses were enrolled and serum HE4 and CA125 were measured in each individual. Modified cut-off values were obtained by maximizing area under the curve (AUC) values and adjusted by using logistic regression with corresponding sensitivity (SN), specificity (SP), Youden index (YI), positive predictive value (PPV), and negative predictive value (NPV).

Results: By utilizing the ideal PPV, NPV, and AUC values, in premenopausal women modified HE4, CA125, ROMA, and PP cut-off values were $73.87 \mathrm{pmol} / \mathrm{L}, 61.60 \mathrm{U} / \mathrm{mL}, 18.47 \%$, and 0.168, respectively. The same test values for postmenopausal women were $120.90 \mathrm{pmol} / \mathrm{L}, 76.21 \mathrm{U} / \mathrm{mL}, 26.48 \%$, and 0.485 , respectively. The $\mathrm{SN}$ for $\mathrm{HE} 4$ with the modified cut-off value was significantly lower than that for CA125 $(\mathrm{P}=0.040)$ in premenopausal women and lower than that for ROMA $(\mathrm{P}=0.001)$ and $\mathrm{PP}(\mathrm{P}=0.044)$ in postmenopausal women. The AUC values for CA125, ROMA, and PP were all significantly higher than that for HE4 ( $\mathrm{P}=0.006,0.007$, and 0.002 , respectively) in postmenopausal women.

Conclusions: The modified cut-off values for HE4, CA125, ROMA, and PP with ideal SN, SP, YI, NPV, PPV were useful of ruling out ovarian malignancy among both pre- and post-menopausal women. In premenopausal women modified HE4, CA125, ROMA, and PP cut-off values were 73.87 pmol/L, 61.60 $\mathrm{U} / \mathrm{mL}, 18.47 \%$, and 0.168 , respectively and in postmenopausal women were $120.90 \mathrm{pmol} / \mathrm{L}, 76.21 \mathrm{U} / \mathrm{mL}$, $26.48 \%$, and 0.485 , respectively.

Keywords: Ovarian tumor (OC); cut-off values; predicted probability model

Submitted Sep 07, 2021. Accepted for publication Oct 29, 2021.

doi: 10.21037 /gs-21-666

View this article at: https://dx.doi.org/10.21037/gs-21-666

\section{Introduction}

Worldwide, ovarian cancer (OC) is one of the most common causes of cancer-related deaths from gynecological neoplasia, and has a 5 -year survival rate of less than $30 \%$ (1). Ovarian cancer symptoms are vague, similar to those of other benign adnexal disorders, and it is often associated with missed diagnosis rates of up to $70 \%$. Siegel reported that approximately $2 / 3$ of all new cases diagnosed with OC resulted death in 2014 (2). In China, the crude incidence rate of OC was 7.95 per 100,000 women with less than $25 \%$ having definite diagnosis (3). Morbidity and mortality are much higher for OC, thus correctly diagnosing potentially malignant ovarian tumors (OTs) is of monumental 
importance in optimizing treatment. In China, while benign OT can be managed at local hospitals, suspected OC should be treated at gynecological centers by expert oncologists for optimal outcomes (4). While clinical examination and imaging modalities are effective in detecting pelvic masses, they lack adequate sensitivity and specificity for distinguishing between benign and malignant tumors.

Biomarkers are powerful, convenient, and non-invasive investigative tools for clinicians to improve the diagnosis and prognosis of OC patients. Cancer antigen 125 (CA125) has long been the most extensively documented circulating biomarker and the most widely-used in distinguishing benign from malignant OTs (5). Elevated serum CA125 levels are found in several benign gynecological diseases such as ovarian endometriosis, leiomyoma, and pelvic infections, and in disorders of other origins, such as liver or renal failure (6), thus it is usually used in combination with other predictors for OC patients.

Human epididymis protein 4 (HE4) is an additional serum biomarker that may be overexpressed in OC (7). It is also expressed in a variety of tissues such as in pulmonary, endometrial, breast adenocarcinomas, and mesotheliomas. Studies reporting on CA125 and HE4 performances in differential benign, or malignant OTs, have been controversial and resulted in inconsistent conclusions (8). The risk of ovarian malignancy algorithm (ROMA) combines HE4 with CA125 measurements into an algorithm based upon menopausal status and has been recommend to classify patients with OT as either low- or high-risk (9).

Some studies have reported that ROMA yields more accurate predictions of the presence of malignant OTs than either CA125 or HE4 alone $(10,11)$ while others have reported no benefits from using either CA125 or HE4 in calculating OC risk (12). There is controversy in the literature regarding the cut-off values for HE4, CA125, and ROMA in differentiating between benign and malignant OTs $(13,14)$. The majority of prior CA125, HE4, and ROMA studies have involved Caucasian or other populations; to date, there have been no unique calculations of predicted probability $(\mathrm{PP})$ risk specifically for Chinese populations. And in clinical application, we found that the cut-off value on the instructions could not distinguish the benign and malignant ovarian tumors for Chinese populations well. Our study aimed to propose new modified HE4, CA125, ROMA, and PP cut-off values, PP calculation model, and evaluate the predictive power of these modified cut-off values in order to distinguish benign from malignant OTs in a Chinese population. In our study, the PP was calculated using logistic regression analysis according to CA125, HE4, ROMA, and menopausal status. Serum HE4 and CA125 were measured, and ROMA and PP were calculated using logistic regression analysis. The cut-off values were modified by logistic regression with corresponding sensitivity (SN), specificity (SP), Youden index (YI), positive predictive value (PPV), negative predictive value (NPV), and AUC values and compared to identify differing OT. We present the following article in accordance with the STARD reporting checklist (available at https://dx.doi.org/10.21037/gs-21-666).

\section{Methods}

\section{Materials}

This was a prospective study conducted at the Third Xiangya Hospital, a gynecology center with oncological expertise and a nationwide referral base. All procedures performed in this study involving human participants were in accordance with the Declaration of Helsinki (as revised in 2013). The study was approved of by the Ethical Committee of the Third Xiangya Hospital (No. 2018-S355) and informed consent was taken from all the patients. We recruited a total of 671 patients who had been diagnosed with OT either by ultrasound, computed tomography (CT), positron emission tomography-computed tomography (PET-CT), or magnetic resonance imaging (MRI) between September 2018 and May 2020. We excluded 137 patients due to non-ovarian active cancer, history of chemotherapy or radiotherapy, disease of the heart, liver, or kidney, or diagnosis of diabetes. The remaining 534 pre- and postmenopausal women with imaging diagnoses of OT were divided into 2 groups based on menopausal status. This was defined as the absence of menstrual periods for 12 months or age greater than 55 years. The OT participants had their tumors surgically removed and specimen samples evaluated by 2 or more gynecological pathologists. Each diagnosis was evaluated and classified as either benign or malignant. Prior to surgery, each participant had blood samples $(5 \mathrm{~mL})$ collected by clinical lab doctors. Samples were centrifuged and fractionated into serum, then the sera was recovered and stored at $-80{ }^{\circ} \mathrm{C}$ until analysis. The concentrations of HE4 and CA125 were measured by a Roche Elecsys Cobas e411 analyzer (Roche Diagnostics, Basel, Switzerland) using the electrochemiluminescence immunoassay (ECLIA) technique. Detection ranges were $15.0-1,500 \mathrm{pmol} / \mathrm{L}$ and 0.600-5,000 U/mL, respectively. All tests were conducted 
based on standard protocols. The ROMA scores were calculated using a logistic regression analysis, as follows:

Premenopausal women, $\mathrm{PI}=-12.0+2.38 \times \mathrm{LN}$ [HE4] $+0.0626 \times \mathrm{LN}$ [CA125]; postmenopausal women, $\mathrm{PI}=-8.09+1.04 \times \mathrm{LN}[\mathrm{HE} 4]+0.732 \times \mathrm{LN}[\mathrm{CA} 125]$; and $\operatorname{ROMA}(\%)=\exp (\mathrm{PI}) /[1+\exp (\mathrm{PI})] \times 100$.

\section{Statistical analysis}

All data materials were analyzed using the SPSS 19.0 (Statistical Package for the Social Sciences, IBM Corp., Chicago, IL, USA). Continuous variables were expressed as mean $\pm \mathrm{SD}$, median and range; independent sample $t$-test and Mann-Whitney $U$ test were used for difference comparisons, and $P$ value $<0.05$ was considered statistically significant. Qualitative data was expressed in percentages. The chisquare test was used for between-group comparisons. The standard of difference significance was $\mathrm{P}=0.05 / \sigma=0.0083$.

The $\mathrm{PP}$ was calculated using logistic regression analysis according to CA125, HE4, ROMA, and menopausal status. Serum HE4 and CA125 were measured, and ROMA and PP were calculated using logistic regression analysis. The cut-off values were modified by logistic regression with corresponding sensitivity (SN), specificity (SP), Youden index (YI), positive predictive value (PPV), negative predictive value (NPV), and AUC values and compared to identify differing OT.

\section{Results}

\section{Clinical characteristics}

The pathology samples were tested after surgery. Each diagnosis was evaluated and classified as either benign $(n=398)$ or malignant $(n=136)$. There were 342 epithelial benign OTs $(85.9 \%, 342 / 398)$ and 125 epithelial OCs (91.9\%, 125/136). The mean participant ages were $36.7 \pm 15.0$ (benign) and 51.0 \pm 13.6 (malignant). In the postmenopausal group, $20.6 \%$ of the participants were benign and $59.8 \%$ were malignant. The OC participants had significantly higher mean ages, menopausal status, and the mean or median levels of CA125 and HE4 as well as the ROMA values $(\mathrm{P}<0.001)$. Clinical characteristics of participants are displayed in Table 1.

\section{HE4, CA125, and ROMA cut-off values specific to the Chinese population}

This study proposes new HE4, CA125, and ROMA cut- off values specific to the Chinese population using a receiver operating characteristic (ROC) analysis. When the AUC values maximized, the ideal cut-off values for $\mathrm{HE} 4$, CA125, and ROMA were calculated. In pre-menopausal participants, AUC [95\% confidence interval (CI)] values were 0.845 ( 0.777 to 0.913$), 0.915$ (0.858 to 0.971$)$, and 0.901 ( 0.850 to 0.951$)$, respectively, and corresponding new cut-off values for HE4, CA125, and ROMA were $73.87,61.60$, and 18.47 , respectively. In post-menopausal participants, AUC $(95 \% \mathrm{CI})$ values were 0.815 (0.746 to 0.883 ), 0.933 (0.897 to 0.968$)$, and 0.931 (0.891 to 0.971 ), respectively, and corresponding new cut-off values for HE4, CA125, and ROMA were 120.90, 76.21, and 26.48, respectively.

\section{PP values calculation formula}

The PP value using CA125, HE4, and ROMA was calculated using the following formula:

Pre-menopausal PP=EXP $(-4.364+0.0003 * \mathrm{HE} 4+0.026$ $\left.6 * \mathrm{CA} 125+0.0398^{*} \mathrm{ROMA}\right) /[1+\mathrm{EXP}(-4.364+0.0003 * \mathrm{HE} 4+$ $\left.\left.0.0266^{*} \mathrm{CA} 125+0.0398^{*} \mathrm{ROMA}\right)\right]$. A PP-score threshold of $\geq 0.168$ indicated a great risk of OC.

Post-menopausal PP=EXP $\left(-3.217-0.0015^{*} \mathrm{HE} 4+0.017\right.$ $\left.1{ }^{*} \mathrm{CA} 125+0.0586^{*} \mathrm{ROMA}\right) /\left[1+\mathrm{EXP}\left(-3.217-0.0015^{*} \mathrm{HE} 4+\right.\right.$ $\left.\left.0.0171^{*} \mathrm{CA} 125+0.0586^{*} \mathrm{ROMA}\right)\right]$. A PP-score threshold of $\geq 0.485$ indicated a great risk of OC.

\section{HE4, CA125, ROMA, and PP diagnostic performances}

The diagnostic performances of HE4, CA125, ROMA, and $\mathrm{PP}$ in distinguishing benign from malignant $\mathrm{OTs}$ were compared using SN, SP, YI, PPV, and NPV. In premenopausal participants: the CA125 SN (85.2\%) was higher than that of HE4 (68.5\%), (P=0.040); the PPV values for HE4, CA125, ROMA, and PP were 63.8\%, 68.6\%, 74.1\%, and $80.0 \%$ respectively, and the NPV values for HE4, CA125, ROMA, and PP were 94.6\%, 97.4\%, 95.6\%, and $96.8 \%$, respectively. The CA125 biomarkers showed a slightly higher NPV and PPV than HE4; whereas, the PP showed higher NPV and PPV than ROMA. The betweengroup comparisons in pre-menopausal participants had no significantly statistical difference, but the SN (81.5\%), SP (96.5\%), YI (0.780), PPV (80.0\%), NPV (96.8\%), and AUC (0.927) for PP were slightly higher than for those HE4, CA125, and ROMA.

In post-menopausal participants, the ROMA SN (90.2\%) was significantly higher than that of HE4 (69.5\%) 


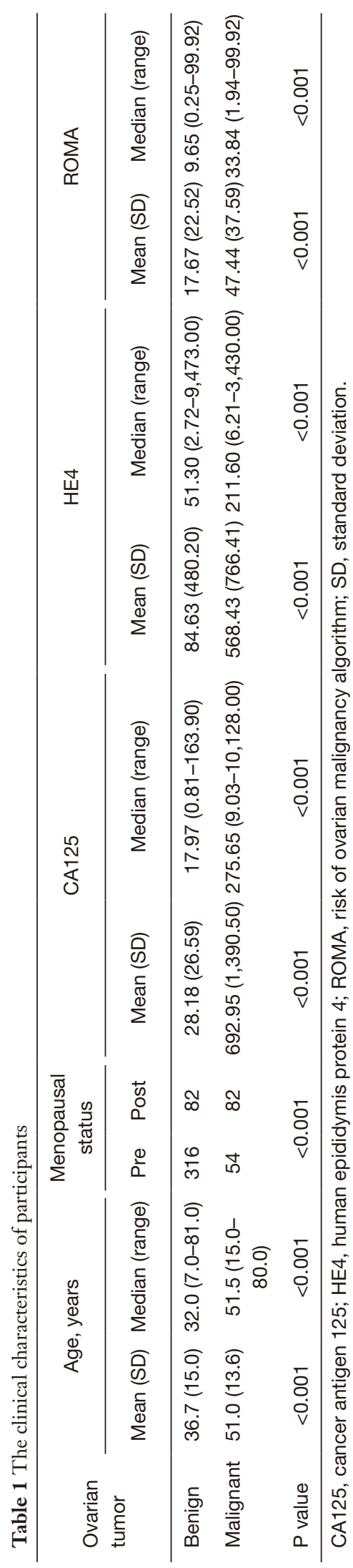

( $\mathrm{P}=0.001)$; the PPV values for HE4, CA125, ROMA, and PP were $87.7 \%, 89.2 \%, 85.1 \%$, and $91.9 \%$, respectively, and NPV values for HE4, CA125, ROMA, and PP were $74.7 \%, 82.2 \%, 89.6 \%$, and $84.4 \%$, respectively. The NPV for ROMA was significantly higher than for HE4 $(\mathrm{P}=0.012)$. The AUC values for CA125 (0.933), ROMA (0.931), and PP (0.949) were significantly higher than that for HE4 (0.815) $(\mathrm{P}=0.006,0.007$, and 0.002 , respectively). The SN (82.9\%), SP (92.7\%), YI (0.756), PPV (91.9\%), NPV (84.4\%), and AUC (0.949) for PP were slightly higher than those for HE4, CA125, and ROMA. The values of HE4, CA125, ROMA, and PP, and their corresponding ROC curves appear in Figures 1,2. The SN, SP, YI, PPV, NPV, and AUC values for HE4, CA125, ROMA, and PP appear in Table 2. The between-group comparisons results appear in Table 3.

\section{Discussion}

As leading cause of death from gynecological malignancies, OC has a dismal prognosis if detected at an advanced stage. The key factors for improving survival rates of OC are an accurate diagnostic approach that detects $\mathrm{OC}$ as soon as possible, followed by correct first surgery (15). All 3 of CA125, HE4, and ROMA have been used for the assessment of OC risk (16). Most prior CA125, HE4, and ROMA studies have involved epithelial OTs as HE4 is mostly significant for epithelial tumors. Although epithelial OTs comprise $50-70 \%$ of primary OTs and $85-90 \%$ of OC, it does not represent all subtypes. Clinically, gynecologists cannot distinguish epithelial OTs from other subtypes; thus, our study and analysis included borderline OTs, non-epithelial OTs, and recurrent OC. It was reported that HE4 expression was not found in borderline OTs and CA125, HE4, and ROMA were not reliable biomarkers in preoperative assessment for borderline OTs (17). Thus, it was considered that inclusion of borderline OTs into the benign group did not affect the performance of CA125 and HE4 in this study. As borderline OTs are generally managed similarly to benign OTs, it was acceptable for borderline OTs to be classified as low risk OTs. Our study had slightly younger mean ages, $36.7 \pm 15.0$ for benign and $51.0 \pm 13.6$ for malignant tumors, in the Chinese population than those previously reported in non-Chinese populations. The proportion of malignancies in all OTs were $15.4 \%$ (premenopausal) and $46.9 \%$ (postmenopausal), which suggests that malignant OTs are mostly found in post-menopausal women and OT 


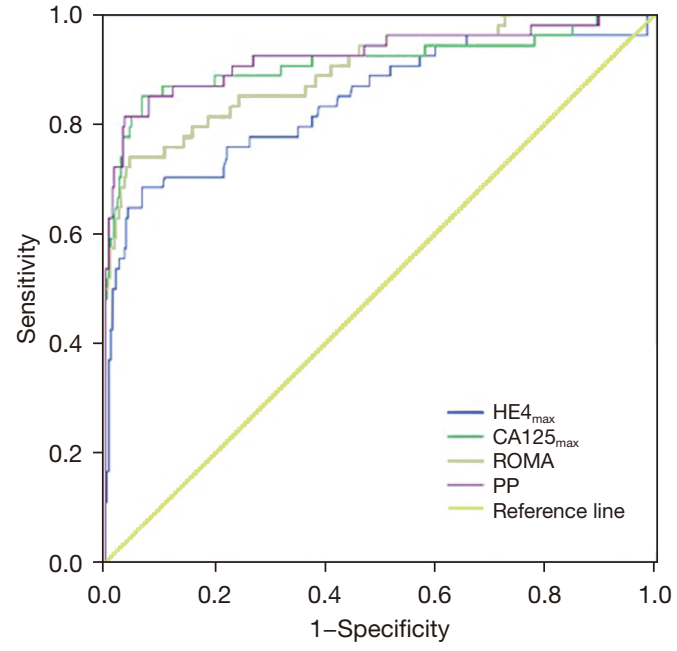

Figure 1 ROC curves for HE4, CA125, ROMA and PP in premenopausal patients. ROC, receiver operating characteristic; CA125, cancer antigen 125; HE4, human epididymis protein 4; ROMA, risk of ovarian malignancy algorithm; PP, predictive probability.

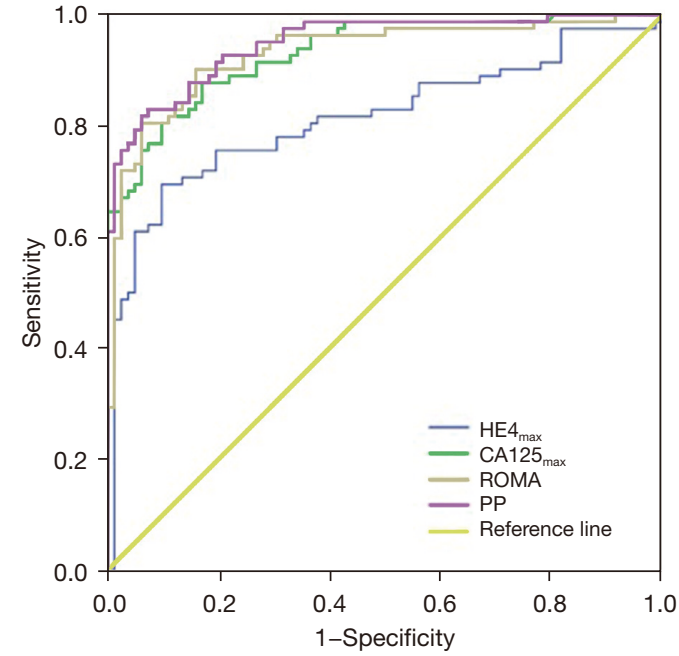

Figure 2 ROC curves for HE4, CA125, ROMA and PP in postmenopausal patients. ROC, receiver operating characteristic; CA125, cancer antigen 125; HE4, human epididymis protein 4; ROMA, risk of ovarian malignancy algorithm; PP, predictive probability.

Table 2 The diagnostic performances of HE4, CA125, ROMA, and PP (benign vs. malignant ovarian tumor)

\begin{tabular}{lccccccc}
\hline Variates & Cut-off value & SN (\%) & SP (\%) & YI & PPV (\%) & NPV (\%) & AUC (95\% CI) \\
\hline Premenopausal $(n=370)$ & & & & & & & \\
HE4 & 73.87 & $68.5(37 / 54)$ & $93.4(295 / 316)$ & 0.619 & $63.8(37 / 58)$ & $94.6(295 / 312)$ & $0.845(0.777,0.913)$ \\
CA125 & 61.60 & $85.2(46 / 54)$ & $93.4(295 / 316)$ & 0.786 & $68.6(46 / 67)$ & $97.4(295 / 303)$ & $0.915(0.858,0.971)$ \\
ROMA & 18.47 & $74.1(40 / 54)$ & $95.6(302 / 316)$ & 0.697 & $74.1(40 / 54)$ & $95.6(302 / 316)$ & $0.901(0.850,0.951)$ \\
PP & 0.168 & $81.5(44 / 54)$ & $96.5(305 / 316)$ & 0.780 & $80.0(44 / 55)$ & $96.8(305 / 315)$ & $0.927(0.878,0.977)$ \\
Post-menopausal (n=164) & & & & & & & \\
HE4 & 120.90 & $69.5(57 / 82)$ & $90.2(74 / 82)$ & 0.597 & $87.7(57 / 65)$ & $74.7(74 / 99)$ & $0.815(0.746,0.883)$ \\
CA125 & 76.21 & $80.5(66 / 82)$ & $90.2(74 / 82)$ & 0.707 & $89.2(66 / 74)$ & $82.2(74 / 90)$ & $0.933(0.897,0.968)$ \\
ROMA & 26.48 & $90.2(74 / 82)$ & $84.1(69 / 82)$ & 0.744 & $85.1(74 / 87)$ & $89.6(69 / 77)$ & $0.931(0.891,0.971)$ \\
PP & 0.485 & $82.9(68 / 82)$ & $92.7(76 / 82)$ & 0.756 & $91.9(68 / 74)$ & $84.4(76 / 90)$ & $0.949(0.919,0.980)$ \\
\hline
\end{tabular}

CA125, cancer antigen 125; HE4, human epididymis protein 4; ROMA, risk of ovarian malignancy algorithm; PP, predicted probability; AUC, area under the curve; $\mathrm{Cl}$, confidence interval.

patients should be divided based on menopausal status. The manufacturer recommended cut-off values of $35 \mathrm{U} / \mathrm{mL}$ for $\mathrm{CA} 125,70 \mathrm{pmol} / \mathrm{L}$ in premenopausal, and $140 \mathrm{pmol} / \mathrm{L}$ in postmenopausal for HE4 could not distinguish benign from malignant OTs accurately as it did not take menopausal status into account (13). The previous studies incorporating standard manufacturer cutoff values resulted in different
SN, SP, and accuracy. Different ethnic populations and genic spectrums can influence the expression rates of biomarkers which may result in different cut-off values. Our study sought to calculate and establish new modified cut-off values for CA125, HE4, and ROMA by a logistic regression analysis depending on menopausal status for the Chinese population. A PP prediction model was calculated using 
Table 3 The $\mathrm{P}$ values of between-group comparisons

\begin{tabular}{|c|c|c|c|c|c|}
\hline Variates & SN (\%) & SP (\%) & PPV (\%) & NPV (\%) & $\mathrm{AUC}(95 \% \mathrm{Cl})$ \\
\hline HE4 vs. CA125 & 0.040 & 1.000 & 0.566 & 0.078 & 0.102 \\
\hline HE4 vs. ROMA & 0.523 & 0.223 & 0.241 & 0.556 & 0.190 \\
\hline HE4 vs. PP & 0.120 & 0.070 & 0.056 & 0.161 & 0.055 \\
\hline CA125 vs. PP & 0.606 & 0.070 & 0.156 & 0.693 & 0.779 \\
\hline ROMA vs. PP & 0.355 & 0.540 & 0.462 & 0.410 & 0.543 \\
\hline \multicolumn{6}{|c|}{ Post-menopausal ( $n=164)$} \\
\hline HE4 vs. CA125 & 0.105 & 1.000 & 0.783 & 0.213 & 0.006 \\
\hline CA125 vs. ROMA & 0.077 & 0.243 & 0.438 & 0.175 & 0.963 \\
\hline CA125 vs. PP & 0.686 & 0.576 & 0.574 & 0.689 & 0.708 \\
\hline ROMA vs. PP & 0.169 & 0.088 & 0.180 & 0.325 & 0.674 \\
\hline
\end{tabular}

SN, sensitivity; SP, specificity; PPV, positive predictive value; NPV, negative predictive value; CA125, cancer antigen 125; HE4, human epididymis protein 4; ROMA, risk of ovarian malignancy algorithm.

logistic regression analysis depending on CA125, HE4, ROMA, and menopausal status.

The modified cut-off value for HE4 was $73.87 \mathrm{pmol} / \mathrm{L}$ in pre-menopausal patients and was close to the manufacturer recommended cut-off value of $70 \mathrm{pmol} / \mathrm{L}$, and it was $120.90 \mathrm{pmol} / \mathrm{L}$ in post-menopausal patients which was lower than the recommended cut-off value. A possible explanation is that HE4 mainly expresses in epithelial OC while this study included $8.09 \%$ (11/136) non-epithelial OC (18). The modified cut-off values for CA125 were $61.60 \mathrm{pmol} / \mathrm{L}$ in pre- and $76.21 \mathrm{pmol} / \mathrm{L}$ in post-menopausal women which were higher than the recommended $35 \mathrm{pmol} / \mathrm{L}$, but the difference between pre- and post-menopausal was minimal. This could be attributed to the fact that CA125 is falsely elevated in patients with benign ovarian cysts and endometriosis (19). The modified cut-off values for ROMA in pre-menopausal patients was $18.47 \%$ and in postmenopausal patients was $26.48 \%$, which were close to the recommended cut-off value. The AUC of HE4, CA125, ROMA, PP in pre-menopausal and post-menopausal patients have high diagnostic value to distinguish benign from malignant OT (AUC >0.7).

Prior studies have found that HE4 is better at differentiating between benign and malignant OT than
CA125 (20). Using the new modified cut-off values, HE4 sensitivity in pre-menopausal $(68.5 \%)$ and postmenopausal women $(69.5 \%)$ was less than for CA125 (85.2\% and $80.5 \%$, respectively), whereas the SP was the same. The PPV refers to the proportion of actual OC cases in the total positive cases detected by screening test, which reflects the diagnostic accuracy of OC. The NPV refers to the proportion of actual non-OC cases in the total negative cases detected by screening test, which reflects the diagnostic accuracy of non-OC. The PPV and NPV for HE4 was less than that for CA125 in this study. The maximum AUCs of HE4 in pre- and post-menopausal women were less than those of CA125. There are several possible reasons for the differences between our study and the other studies, including: (I) there were more OT patients $(n=534)$ in our study than in most of the other studies (3); (II) participants with low malignant potential had low HE4 levels (21); (III) subtype percentages can affect results (non-epithelial OTs included in our study: 56 non-epithelial benign OTs and 11 epithelial OCs; clear cell carcinomas and mucinous tumors included in our study did not express HE4 (22); and (IV) menopausal status and surgical stage may greatly effect HE4 levels (23).

The PP, calculated by logistic regression algorithm, has a 
higher diagnostic accuracy than HE4, CA125, and ROMA, showing a higher SN, SP, YI, PPV, NPV, and AUC in preand post-menopausal patients.

In the validation data sets with new modified cutoff values, HE4, CA125, ROMA, and PP had the same statistical differences in SN, SP, YI, PPV, NPV, and AUC values. There was a slight difference in the results of the different malignancy percentages. The AUC values in validation data sets were all above 0.7 , which demonstrated the modified cut-off values had high diagnostic value for distinguishing benign from malignant OT. Since the validation data sets were much smaller than the development data sets, the diagnostic performances of validation data sets were poorer than those of the development data sets. There were no significant differences between the validation data sets and the development data sets $(\mathrm{P}>0.01)$.

In conclusion, the modified cut-off values for HE4, CA125, ROMA, and PP for Chinese populations at risk of OC can increase diagnostic accuracy according to menopausal status by utilizing the parameters recommended above. Similar results were demonstrated by Sturgeon et al. (24). What's more, compared to Xu et al. (14) determined cut-off value, the modified cut-off values for HE4, CA125, ROMA in our study performance better in differentiating benign from malignant. In our study, CA125 was found to be an excellent biomarker when used alone to differentiate benign from malignant pelvic masses which means we should pay more attention to the result of CA125. In light of the results of this study, a PP algorithm has the greatest diagnostic value in cases of incidental detection of a pelvic tumor. This may help physicians in primary care settings as it provides an objective evaluation of malignancy risk and may aid in optimizing treatments for those with either a benign or malignant OT. Additional, imaging examination is equally important to differentiate ovarian tumor benign and malignant. Benign OTs can be managed at local hospitals which reduces overall costs incurred by referrals and transfers. Suspected OC should be treated at gynecological centers with oncologic expertise which results in improved rates of first-surgical success and 5-year survival. Particularly during the COVID-19 epidemic, the differential diagnosis of benign and malignant OT is very important. Patients with benign tumors can postpone treatment or receive local treatment, while those with malignant tumors should actively take protective measures and contact superior hospitals for further diagnosis and treatment.

\section{Acknowledgments}

We thank Shanghai Labway Clinical Laboratory Co., Ltd. for their technical assistance.

Funding: This study was supported by the Natural Science Foundation of Hunan Province (2020JJ5872), the Hunan Provincial Clinical Medical Technology Innovation Guiding Project (2020SK53606), the Hunan Science and Technology Department (Grant 2020 SK4017), and National Key Research and Development Program of China (2018YFC1004800).

\section{Footnote}

Reporting Checklist: The authors have completed the STARD reporting checklist. Available at https://dx.doi. org/10.21037/gs-21-666

Data Sharing Statement: Available at https://dx.doi. org/10.21037/gs-21-666

Conflicts of Interest: All authors have completed the ICMJE uniform disclosure form (available at https://dx.doi. org/10.21037/gs-21-666). All authors report this study was supported by the Natural Science Foundation of Hunan Province (2020JJ5872), the Hunan Provincial Clinical Medical Technology Innovation Guiding Project (2020SK53606), the Hunan Science and Technology Department (Grant 2020 SK4017), and National Key Research and Development Program of China (2018YFC1004800) and received technical assistance Shanghai Labway Clinical Laboratory Co., Ltd. The authors have no other conflicts of interest to declare.

Ethical Statement: The authors are accountable for all aspects of the work in ensuring that questions related to the accuracy or integrity of any part of the work are appropriately investigated and resolved. All procedures performed in this study involving human participants were in accordance with the Declaration of Helsinki (as revised in 2013). The study was approved of by the Ethical Committee of the Third Xiangya Hospital (No. 2018-S355) and informed consent was taken from all the patients.

Open Access Statement: This is an Open Access article distributed in accordance with the Creative Commons Attribution-NonCommercial-NoDerivs 4.0 International 
License (CC BY-NC-ND 4.0), which permits the noncommercial replication and distribution of the article with the strict proviso that no changes or edits are made and the original work is properly cited (including links to both the formal publication through the relevant DOI and the license). See: https://creativecommons.org/licenses/by-nc-nd/4.0/.

\section{References}

1. Funston G, Hardy V, Abel G, et al. Identifying Ovarian Cancer in Symptomatic Women: A Systematic Review of Clinical Tools. Cancers (Basel) 2020;12:3686.

2. Siegel R, Ma J, Zou Z, et al. Cancer statistics, 2014. CA Cancer J Clin 2014;64:9-29.

3. Zhang P, Wang C, Cheng L, et al. Comparison of HE4, CA125, and ROMA Diagnostic Accuracy: A Prospective and Multicenter Study for Chinese Women With Epithelial Ovarian Cancer. Medicine (Baltimore) 2015;94:e2402.

4. Chen Z, Liang Q, Zeng H, et al. Exosomal CA125 as A Promising Biomarker for Ovarian Cancer Diagnosis. J Cancer 2020;11:6445-53.

5. Ledermann JA, Raja FA, Fotopoulou C, et al. Newly diagnosed and relapsed epithelial ovarian carcinoma: ESMO Clinical Practice Guidelines for diagnosis, treatment and follow-up. Ann Oncol 2013;24 Suppl 6:vi24-32.

6. Park Y, Lee JH, Hong DJ, et al. Diagnostic performances of HE 4 and CA125 for the detection of ovarian cancer from patients with various gynecologic and nongynecologic diseases. Clin Biochem 2011;44:884-8.

7. Moore RG, Brown AK, Miller MC, et al. The use of multiple novel tumor biomarkers for the detection of ovarian carcinoma in patients with a pelvic mass. Gynecol Oncol 2008;108:402-8.

8. Yang $Z$, Wei C, Luo Z, et al. Clinical value of serum human epididymis protein 4 assay in the diagnosis of ovarian cancer: a meta-analysis. Onco Targets Ther 2013;6:957-66.

9. Van Gorp T, Cadron I, Despierre E, et al. HE4 and CA125 as a diagnostic test in ovarian cancer: prospective validation of the Risk of Ovarian Malignancy Algorithm. Br J Cancer 2011;104:863-70.

10. Ikeda Y, Hasegawa K, Kurosaki A, et al. The Risk of Ovarian Malignancy Algorithm (ROMA) as a Predictive Marker of Peritoneal Dissemination in Epithelial Ovarian Cancer Patients. Oncol Res Treat 2015;38:276-81.

11. Romagnolo C, Leon AE, Fabricio ASC, et al. HE4,
CA125 and risk of ovarian malignancy algorithm (ROMA) as diagnostic tools for ovarian cancer in patients with a pelvic mass: An Italian multicenter study. Gynecol Oncol 2016;141:303-11.

12. Wang J, Gao J, Yao H, et al. Diagnostic accuracy of serum HE4, CA125 and ROMA in patients with ovarian cancer: a meta-analysis. Tumour Biol 2014;35:6127-38.

13. Chen X, Zhou H, Chen R, et al. Development of a multimarker assay for differential diagnosis of benign and malignant pelvic masses. Clin Chim Acta 2015;440:57-63.

14. Xu Y, Zhong R, He J, et al. Modification of cut-off values for HE4, CA125 and the ROMA algorithm for early-stage epithelial ovarian cancer detection: Results from 1021 cases in South China. Clin Biochem 2016;49:32-40.

15. Sun H, Yan L, Chen H, et al. Development of a nomogram to predict prognosis in ovarian cancer: a SEER-based study. Transl Cancer Res 2020;9:5829-42.

16. Al Musalhi K, Al Kindi M, Al Aisary F, et al. Evaluation of HE4, CA-125, Risk of Ovarian Malignancy Algorithm (ROMA) and Risk of Malignancy Index (RMI) in the Preoperative Assessment of Patients with Adnexal Mass. Oman Med J 2016;31:336-44.

17. Gizzo S, Berretta R, Di Gangi S, et al. Borderline ovarian tumors and diagnostic dilemma of intraoperative diagnosis: could preoperative He4 assay and ROMA score assessment increase the frozen section accuracy? A multicenter casecontrol study. Biomed Res Int 2014;2014:803598.

18. Chan KK, Chen CA, Nam JH, et al. The use of HE4 in the prediction of ovarian cancer in Asian women with a pelvic mass. Gynecol Oncol 2013;128:239-44.

19. Holcomb K, Vucetic Z, Miller MC, et al. Human epididymis protein 4 offers superior specificity in the differentiation of benign and malignant adnexal masses in premenopausal women. Am J Obstet Gynecol 2011;205:358.e1-6.

20. Cho HY, Park SH, Park YH, et al. Comparison of HE4, CA125, and Risk of Ovarian Malignancy Algorithm in the Prediction of Ovarian Cancer in Korean Women. J Korean Med Sci 2015;30:1777-83.

21. Kotowicz B, Fuksiewicz M, Sobiczewski P, et al. Clinical value of human epididymis protein 4 and the Risk of Ovarian Malignancy Algorithm in differentiating borderline pelvic tumors from epithelial ovarian cancer in early stages. Eur J Obstet Gynecol Reprod Biol 2015;194:141-6.

22. Fujiwara H, Suzuki M, Takeshima N, et al. Evaluation of human epididymis protein 4 (HE4) and Risk of Ovarian Malignancy Algorithm (ROMA) as diagnostic tools of type 
I and type II epithelial ovarian cancer in Japanese women. Tumour Biol 2015;36:1045-53.

23. Karlsen MA, Høgdall EV, Christensen IJ, et al. A novel diagnostic index combining HE4, CA125 and age may improve triage of women with suspected ovarian cancer - An international multicenter study in women with an

Cite this article as: Zhao X, Zhao M, Gao B, Zhang A, Xu D. Modified HE4, CA125, and ROMA cut-off values and predicted probability of ovarian tumor in Chinese patients. Gland Surg 2021;10(11):3097-3105. doi: 10.21037/gs-21-666 ovarian mass. Gynecol Oncol 2015;138:640-6.

24. Sturgeon CM, Duffy MJ, Stenman UH, et al. National Academy of Clinical Biochemistry laboratory medicine practice guidelines for use of tumor markers in testicular, prostate, colorectal, breast, and ovarian cancers. Clin Chem 2008;54:e11-79. 